\title{
Dynamics of continuous-time quantum walks in restricted geometries
}

\author{
E. Agliari, A. Blumen and O. Mülken \\ Theoretische Polymerphysik, Universität Freiburg, Hermann-Herder-Str. 3, D-79104 \\ Freiburg, Germany
}

\begin{abstract}
We study quantum transport on finite discrete structures and we model the process by means of continuous-time quantum walks. A direct and effective comparison between quantum and classical walks can be attained based on the average displacement of the walker as a function of time. Indeed, a fast growth of the average displacement can be advantageously exploited to build up efficient search algorithms. By means of analytical and numerical investigations, we show that the finiteness and the inhomogeneity of the substrate jointly weaken the quantum walk performance. We further highlight the interplay between the quantum-walk dynamics and the underlying topology by studying the temporal evolution of the transfer probability distribution and the lower bound of long time averages.
\end{abstract}

PACS numbers: 05.60Gg, 71.35.-y, 05.60Cd 


\section{Introduction}

Quantum walks (QWs) are attracting increasing attention in many research areas, ranging from solid-state physics to quantum computing [1]. In particular, QWs provide a model for quantum-mechanical transport processes on discrete structures; this includes, for instance, the coherent energy transfer of a qubit on an optical lattice [2, 3, 4, 5]. The theoretical study of QWs is also encouraged by recent experimental implementations able to corroborate theoretical findings [6, 7, 8].

As in the classical random walk, quantum walks appear in a discrete [9] as in a continuous-time (CTQW) [10] form; these forms, however, cannot be simply related to each other [11]. Now, standard CTQWs, on which we focus, can be obtained by identifying the Hamiltonian of the system with the classical transfer matrix which is, in turn, directly related to the Laplacian of the underlying structure.

Another feature which CTQWs share with classical random walks consists in the strong interplay between the dynamics properties displayed by the walk and the topology of the substrate [12, 13, 14]. However, the dependencies turn out to be much more complex in the quantum-mechanical case: while the classical (simple) walk eventually loses memory of its starting site, the quantum walk exhibits, even in the asymptotic regime, transition probabilities which depend on the starting site. For this reason, often the parameters describing the transport are averaged over all initial sites, a procedure which allows a global characterization of the walk, while preserving its most important features.

One of the quantities affected by topology is the mean square displacement of the walker up to time $t$. Classically, this quantity is monotonically increasing and depends (asymptotically) on time according to the power law $\left\langle r^{2}(t)\right\rangle \sim t^{\beta}$. The value of the "diffusion exponent" $\beta$ allows to distinguish between normal $(\beta=1)$ and anomalous $(\beta \neq 1)$ diffusion [15]. As for quantum transport, it is possible to introduce analogous exponents, characterizing the temporal spreading of a wave-packet [16]. However, even when they take place over the same structure, quantum and classical walks can exhibit dramatically different behaviours. In particular, the quantum wave propagation on regular, infinite lattices is ballistic, i.e. the root mean square displacement is linear in time. Such a quadratic speed-up of the mean square displacement is a well known phenomenon when dealing with tight-binding electron waves on periodic lattices [9] and, from a computational point of view, it constitutes an important feature since it could be advantageously exploited in quantum search algorithms [17, 18, 19]. In the presence of disorder (either deterministic or stochastic) or finiteness, the sharp ballistic fronts are softened, a fact which may even lead to the localization of the quantum particle [20, 21]. It is therefore of both theoretical and practical interest to highlight how finiteness and inhomogeneity - often unavoidable in real systems - affect the particle's propagation. To this aim we analyze quantum transport on restricted geometries, where the restrictions arise from the (possibly joint) fractal dimension and finite extent of the substrate itself. By direct comparison with the classical case, we find that, on finite 
substrates, the advantage of CTQWs is at short times only. Moreover, the lack of translational invariance weakens the CTQW performance, i.e. in such situations the average displacement increases more slowly with time.

The finite discrete structures we consider and compare are the Dual Sierpinski Gasket (DSG), the Cayley Tree (CT) vide infra Sec. 4, and the square lattice with periodic boundary conditions, i.e. the square torus (ST). These constitute representative topologies, providing examples of fractals with loops, of trees and of regular structures. The Dual Sierpinski Gasket will be treated in more detail; for this structure the eigenvalue spectrum of the Laplacian matrix is known exactly, allowing for some analytical estimates. Indeed, not only random walks, but also many dynamical properties of connected structures themselves (such as the vibrational structures and the relaxation modes) depend on the spectrum of their Laplacian matrix [22]. However, for CTQWs the set of eigenvectors also matters, which often makes analytical investigations cumbersome.

It is worth underlining that focusing on discrete structures is not only suggested by solid-state applications: quantum computation is traditionally concerned with the manipulation of discrete systems. In particular, a discrete (and finite) state space makes the CTQW simulation by quantum computers, working with discrete registers, feasible [1, 23].

Our paper is structured as follows. After a brief summary of the main concepts and of the formulæ concerning CTQWs in Sec. 2, we describe the topology of the DSG in Sec. 3. Then, in Sec. 4, we study the quantum-mechanical transport over the above mentioned structures, especially focusing on the average displacement and on the long time averages. Finally, in Sec. 5 we present our comments and conclusions. In the Appendix we derive analytical results concerning the average chemical displacements of CTQWs over hypercubic lattices, special cases being chains and square lattices.

\section{Continuous-time quantum walks on graphs}

Mathematically, a graph is specified by the pair $\{V, E\}$ consisting of a nonempty, countable set of points $V$, joined pairwise by a set of links $E$. The cardinality of $V$ provides the number $\mathcal{N}$ of sites making up the graph, i.e. its volume: $|V|=\mathcal{N}$. In the following, we focus mainly on finite graphs $(\mathcal{N}<\infty)$ and we label each node with a lowercase letter $i \in V$.

From an algebraic point of view, a graph can be described by its adjacency matrix A, whose elements are

$$
A_{i j}= \begin{cases}1 & \text { if }(i, j) \in E \\ 0 & \text { otherwise }\end{cases}
$$

The connectivity of a node $i$ can be calculated as a sum of matrix elements $z_{i}=\sum_{j} A_{i j}$. The Laplacian operator is then defined as $\mathbf{L}=\mathbf{Z}-\mathbf{A}$, where $\mathbf{Z}$ is the diagonal matrix given by $Z_{i k}=z_{i} \delta_{i k}$. 
The Laplacian matrix $\mathbf{L}$ is symmetric and non-negative definite and it can therefore generate a probability conserving Markov process and define a unitary process as well. Otherwise stated, the Laplacian operator can work both as a classical transfer operator and as a tight-binding Hamiltonian of a quantum transport process [24, 25].

Indeed, the classical continuous-time random walk (CTRW) is described by the following Master equation [26]:

$$
\frac{d}{d t} p_{k, j}(t)=\sum_{l=1}^{\mathcal{N}} T_{k l} p_{l, j}(t),
$$

where $p_{k, j}(t)$ is the conditional probability that the walker is on node $k$ when it started from node $j$ at time 0 . If the walk is symmetric with a site-independent transmission rate $\gamma$, then the transfer matrix $\mathbf{T}$ is simply related to the Laplacian operator through $\mathbf{T}=-\gamma \mathbf{L}$.

Now the CTQW, the quantum-mechanical counterpart of the CTRW, is introduced by identifying the Hamiltonian of the system with the classical transfer matrix, $\mathbf{H}=-\mathbf{T}$ [10, 13, 24] (in the following we will set $\hbar \equiv 1$ ). The set of states $|j\rangle$, representing the walker localized at the node $j$, spans the whole accessible Hilbert space and also provides an orthonormal basis set. Therefore, the behaviour of the walker can be described by the transition amplitude $\alpha_{k, j}(t)$ from state $|j\rangle$ to state $|k\rangle$, which obeys the following Schrödinger equation:

$$
\frac{d}{d t} \alpha_{k, j}(t)=-i \sum_{l=1}^{\mathcal{N}} H_{k l} \alpha_{l, j}(t) .
$$

If at the initial time $t_{0}=0$ only the state $|j\rangle$ is populated, then the formal solution to Eq. 2 can be written as

$$
\alpha_{k, j}(t)=\langle k|\exp (-i \mathbf{H} t)| j\rangle,
$$

whose squared magnitude provides the quantum mechanical transition probability $\pi_{k, j}(t) \equiv\left|\alpha_{k, j}(t)\right|^{2}$. In general, it is convenient to introduce the orthonormal basis $\left|\psi_{n}\right\rangle, n \in[1, \mathcal{N}]$ which diagonalizes $\mathbf{T}$ (and, clearly, also $\mathbf{H}$ ); the correspondent set of eigenvalues is denoted by $\left\{\lambda_{n}\right\}_{n=1, \ldots, \mathcal{N}}$. Thus, we can write

$$
\pi_{k, j}(t)=\left|\sum_{n=1}^{\mathcal{N}}\left\langle k\left|e^{-i \lambda_{n} t}\right| \psi_{n}\right\rangle\left\langle\psi_{n} \mid j\right\rangle\right|^{2} .
$$

Despite the apparent similarity between Eq. 1 and 2, some important differences are worth being recalled.

First of all, the imaginary unit makes the time evolution operator $\mathbf{U}(t)=\exp (-i \mathbf{H} t)$ unitary, which prevents the quantum mechanical transition probability from having a definite limit as $t \rightarrow \infty$. On the other hand, a particle performing a CTRW is asymptotically equally likely to be found on any site of the structure: the classical $p_{k, j}(t)$ admit a stationary distribution which is independent of initial and final sites, $\lim _{t \rightarrow \infty} p_{k, j}(t)=1 / \mathcal{N}$. Hence, in order to compare classical long time probabilities with quantum mechanical ones, we rely on the long time average (LTA) [27], defined in 
Sec. 4.4,

Moreover, the normalization conditions for $p_{k, j}(t)$ and $\alpha_{k, j}(t) \operatorname{read} \sum_{k=1}^{\mathcal{N}} p_{k, j}(t)=1$, and $\sum_{k=1}^{\mathcal{N}}\left|\alpha_{k, j}(t)\right|^{2}=1$

\subsection{Average displacement}

The average displacement performed by a quantum walker until time $t$ allows a straightforward comparison with the classical case; it is also more directly related to transport properties than the transfer probability $\pi_{k, j}(t)$ : It constitutes the expectation value of the distance reached by the particle after a time $t$ and its time dependence provides information on how fast the particle propagates over the substrate.

For CTQW (subscript $q$ ) starting at node $j$, we define the average (chemical) displacement $\left\langle r_{j}(t)\right\rangle_{q}$ performed until time $t$ as

$$
\left\langle r_{j}(t)\right\rangle_{q}=\sum_{k=1}^{\mathcal{N}} \ell(k, j) \pi_{k, j}(t),
$$

where $\ell(k, j)$ is the chemical distance between the sites $j$ and $k$, i.e. the length of the shortest path connecting $j$ and $k$. We can average over all starting points to obtain

$$
{\overline{\langle r(t)\rangle_{q}}}=\frac{1}{\mathcal{N}} \sum_{j=1}^{\mathcal{N}}\left\langle r_{j}(t)\right\rangle_{q} .
$$

For fractals or hyperbranched structures it is more appropriate to use the chemical distance, rather than the Euclidean distance; for instance, the infinite CT (see Sec. 4) cannot be embedded in any lattice of finite dimension. For classical diffusion, it is wellknown that the chemical and the Euclidean distances display analogous asymptotic laws for regular structures and for many deterministic fractals (e.g. the Sierpinski Gasket) [15]; as discussed in the Appendix, this still holds for CTQWs on arbitrary $d$-dimensional hypercubic lattices.

For classical (subscript $c$ ) regular diffusion (on infinite lattices) the average displacement $\langle r(t)\rangle_{c}$ depends on time $t$ according to

$$
\langle r(t)\rangle_{c} \sim t^{1 / 2}
$$

More generally, for scaling (fractal) structures we can define the so-called chemical diffusion exponent $d_{w}^{\ell}$ and get [15]:

$$
\langle r(t)\rangle_{c} \sim t^{1 / d_{w}^{\ell}}
$$

Finite systems require corrections to these laws: for them $\langle r(t)\rangle_{c}$ does not grow indefinitely, but it saturates to a maximum value $r_{c}[28]$.

\subsection{Return Probability}

As it is well known, for a diffusive particle the probability to return to the starting point is topology sensitive, and it can indeed be used to extract information about the 
underlying structure [26]. It is therefore interesting to compare the classical return probability $p_{k, k}(t)$ with the quantum mechanical $\pi_{k, k}(t)$ (see also [29, 30]). One has

$$
p_{k, k}(t)=\langle k|\exp (\mathbf{T} t)| k\rangle=\sum_{n=1}^{\mathcal{N}}\left|\left\langle k \mid \psi_{n}\right\rangle\right|^{2} \exp \left(-\gamma t \lambda_{n}\right)
$$

and

$$
\pi_{k, k}(t)=\left|\alpha_{k, k}(t)\right|^{2}=\left.\left.\left|\sum_{n=1}^{\mathcal{N}}\right|\left\langle k \mid \psi_{n}\right\rangle\right|^{2} \exp \left(-i \gamma t \lambda_{n}\right)\right|^{2} .
$$

In order to get a global information about the likelihood to be (return or stay) at the origin, independent of the starting site, we average over all sites of the graph, obtaining

$$
\bar{p}(t)=\frac{1}{\mathcal{N}} \sum_{k=1}^{\mathcal{N}} p_{k, k}(t)=\frac{1}{\mathcal{N}} \sum_{n=1}^{\mathcal{N}} e^{-\gamma \lambda_{n} t}
$$

and

$$
\bar{\pi}(t)=\frac{1}{\mathcal{N}} \sum_{k=1}^{\mathcal{N}} \pi_{k, k}(t)=\frac{1}{\mathcal{N}} \sum_{n, m=1}^{\mathcal{N}} e^{-i \gamma\left(\lambda_{n}-\lambda_{m}\right) t} \sum_{k=1}^{\mathcal{N}}\left|\left\langle k \mid \psi_{n}\right\rangle\right|^{2}\left|\left\langle k \mid \psi_{m}\right\rangle\right|^{2} .
$$

For finite substrates, the classical $\bar{p}(t)$ decays monotonically to the equipartition limit, and it only depends on the eigenvalues of $\mathbf{T}$. On the other hand, $\bar{\pi}(t)$ depends explicitly on the eigenvectors of $\mathbf{H}[29,30]$. By means of the Cauchy-Schwarz inequality we can obtain a lower bound for $\bar{\pi}(t)$ which does not depend on the eigenvectors [30, 31]:

$$
\bar{\pi}(t) \geq\left|\frac{1}{\mathcal{N}} \sum_{k=1}^{\mathcal{N}} \alpha_{k, k}(t)\right| \equiv|\bar{\alpha}(t)|^{2}=\frac{1}{\mathcal{N}^{2}} \sum_{m, n=1}^{\mathcal{N}} e^{-i \gamma\left(\lambda_{n}-\lambda_{m}\right) t} .
$$

Notice that Eqs. 11 and 12 can serve as measures of the efficiency of the transport process performed by CTRW and CTQW, respectively. In fact, the faster $\bar{p}(t)$ decreases towards its asymptotic value, the more efficient the transport. Analogously, a more rapid decay of the envelope of $\bar{\pi}(t)$ (or of $|\bar{\alpha}(t)|^{2}$ ) implies a faster delocalization of the quantum walker over the graph. By the way, we recall that, for a large variety of graphs [30], the classical average return probability scales as $\bar{p}(t) \sim t^{-\mu}$, while the envelope of $|\bar{\alpha}(t)|^{2}$, namely env $\left[|\bar{\alpha}(t)|^{2}\right]$, scales like $t^{-2 \mu}, \mu$ being a proper parameter related for fractals to the spectral density.

As can be inferred by comparing Eqs. 11 and 12, for quantum transport processes the degeneracy of the eigenvalues plays an important role, as the differences between eigenvalues determine the temporal behaviour, while for classical transport the long time behaviour is dominated by the smallest eigenvalue. Situations in which only a few, highly degenerate eigenvalues are present are related to slow CTQW dynamics, while when all eigenvalues are non-degenerate the transport turns out to be efficient [29, 30]. 


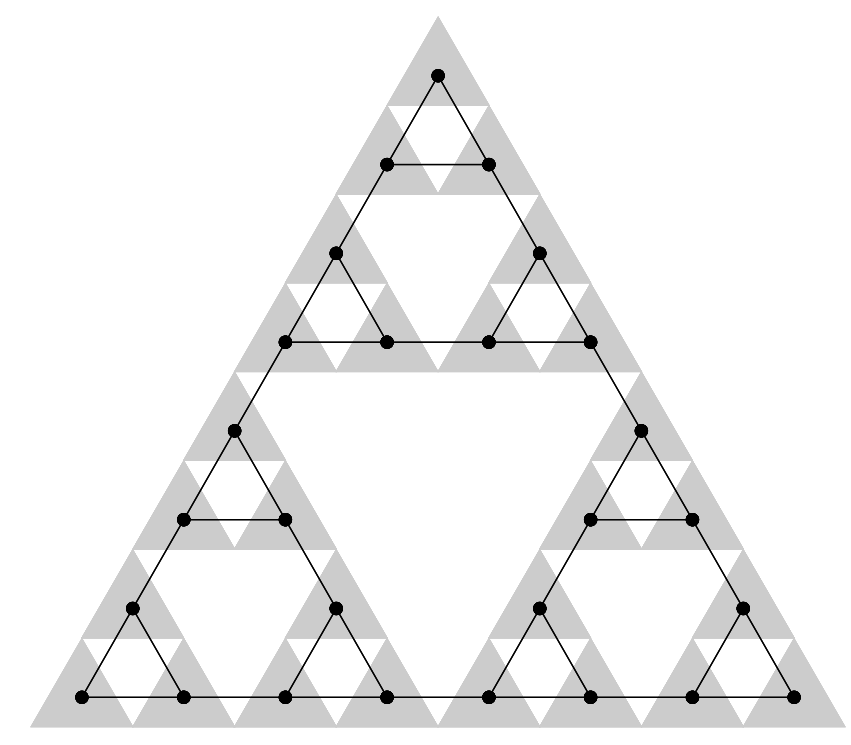

Figure 1. Dual transformation from Sierpinski Gasket to Dual Sierpinski Gasket of generation $g=3$.

\section{Dual Sierpinski gasket: Topology and eigenvalue spectrum}

Before turning to the dynamics of CTQW (and CTRW) on exemplary structures, which allow us to highlight the importance of inhomogeneities, some remarks on the spectra of the DSG are in order.

The dual Sierpinski gasket is an exactly-decimable fractal which is directly related, through a dual transformation, to the Sierpinski gasket (SG). The DSG of generation $g$ can be constructed by replacing each small triangle belonging to the SG with a node and by connecting such nodes whenever the relevant triangles share a vertex in the original gasket (see Fig. 1). It is straightforward to verify that the number of nodes at any given generation $g$ is $\mathcal{N}=3^{g}$.

The dual transformation does not conserve the coordination number of the inner nodes (which decreases from 4 to 3 ), but it does conserve the fractal dimension $d_{f}$ and the spectral dimension $\tilde{d}$, which are therefore the same as for the original Sierpinski gasket $d_{f}=\ln 3 / \ln 2=1.58496 \ldots$ and $\tilde{d}=2 \ln 3 / \ln 5=1.36521 \ldots$.

As mentioned above, the knowledge of the eigenvalue spectrum is sufficient for the calculation of several interesting quantities concerning the dynamics of CTQWs. In general, any (finite) Hamiltonian $\mathbf{H}$ can be (at least numerically) diagonalized in order to obtain its spectrum. However, as the size of $\mathbf{H}$ gets large, the procedure gets to be time consuming and the precise numerical diagonalization may not be easy to perform. 
Remarkably, the eigenvalue spectrum of the DSG Laplacian matrix can be determined at any generation through the following iterative procedure; for more details we refer to [34, 35]: At any given generation $g$ the spectrum includes the non-degenerate eigenvalue $\lambda_{\mathcal{N}}=0$, the eigenvalue 3 with degeneracy $\left(3^{g-1}+3\right) / 2$ and the eigenvalue 5 with degeneracy $\left(3^{g-1}-1\right) / 2$. Moreover, given the eigenvalue spectrum at generation $g-1$, each non-vanishing eigenvalue $\lambda_{g-1}$ corresponds to two new eigenvalues $\lambda_{g}^{ \pm}$according to

$$
\lambda_{g}^{ \pm}=\frac{5 \pm \sqrt{25-4 \lambda_{g-1}}}{2} ;
$$

both $\lambda_{g}^{+}$and $\lambda_{g}^{-}$inherit the degeneracy of $\lambda_{g-1}$. The eigenvalue spectra is therefore bounded in $[0,5]$. As explained in [34], at any generation $g$, we can calculate the degeneracy of each distinct eigenvalue: apart from $\lambda_{\mathcal{N}}$ whose degeneracy is 1 , there are $2^{r}$ distinct eigenvalues, each with degeneracy $\left(3^{g-r-1}+3\right) / 2$, being $r=0,1, \ldots, g-1$, and $2^{r}$ distinct eigenvalues, each with degeneracy $\left(3^{g-r-1}-1\right) / 2$, being $r=0,1, \ldots, g-2$. As can be easily verified, the degeneracies sum up to $\mathcal{N}=3^{g}$. Finally, notice that the distribution of eigenvalues and their degeneracies are non-uniform and that the spectrum is multifractal [34].

\section{CTQWs on restricted geometries}

\subsection{Transfer probability}

Results for the exact transition probability distribution $\pi_{k, j}(t)$ for STs and CTs have already been given in Refs. [13, 31, 32, where it was shown that $\pi_{k, j}(t)$ depends significantly on the starting node. Results for ultrametric structures are given in [33].

It is worth recalling here that the Cayley tree $(\mathrm{CT})$ can be built by starting from one node (root) connected to $z$ nodes, which constitute the first shell. Each node of the first shell is then connected to $z-1$ new nodes, which constitute the second shell and so forth, iteratively. Therefore, the $M$-th shell contains $z(z-1)^{M-1}$ nodes which are at a chemical distance $M$ from the root. Thus, the CT is a $z$-regular loop-free graph. The numbers of sites in a CT of $M$ shells is $\mathcal{N}_{M}=\left[z(z-1)^{M}-2\right] /(z-2)$, hence the correlated fractal dimension $\log \left(\mathcal{N}_{M}\right) / \log (M)$ goes to infinity for $M \rightarrow \infty$, precluding the possibility of embedding very large $\mathrm{CT}$ in any previously specified Euclidean lattice. In the following we focus on finite 3-Cayley trees, which means that $z$ is fixed and equal to three for any internal site of the graph; furthermore, the number of shells (also called generation) is finite (and therefore also the number of nodes is itself finite).

In Fig. 2 we show our results for a DSG of generation $g=3$ and we focus on the set of pairs given by $\left(v_{n}, 1\right)$, where $v_{n}$ denotes any of the two corners of the gasket of the $n$-th generation, with $n \leqslant g$ (i.e., according to the labeling of Fig. 2, $\left.v_{0}=1, v_{1}=2, v_{2}=5, v_{3}=14\right)$. Now, due to the symmetry the DSG is endowed with, for CTQWs starting from a given vertex, say the apex, the left and right corners are equivalent. As expected, $\pi_{k, j}(t)$ does not converge to any definite value, but it displays oscillations whose amplitudes and average values get smaller as the distance between 


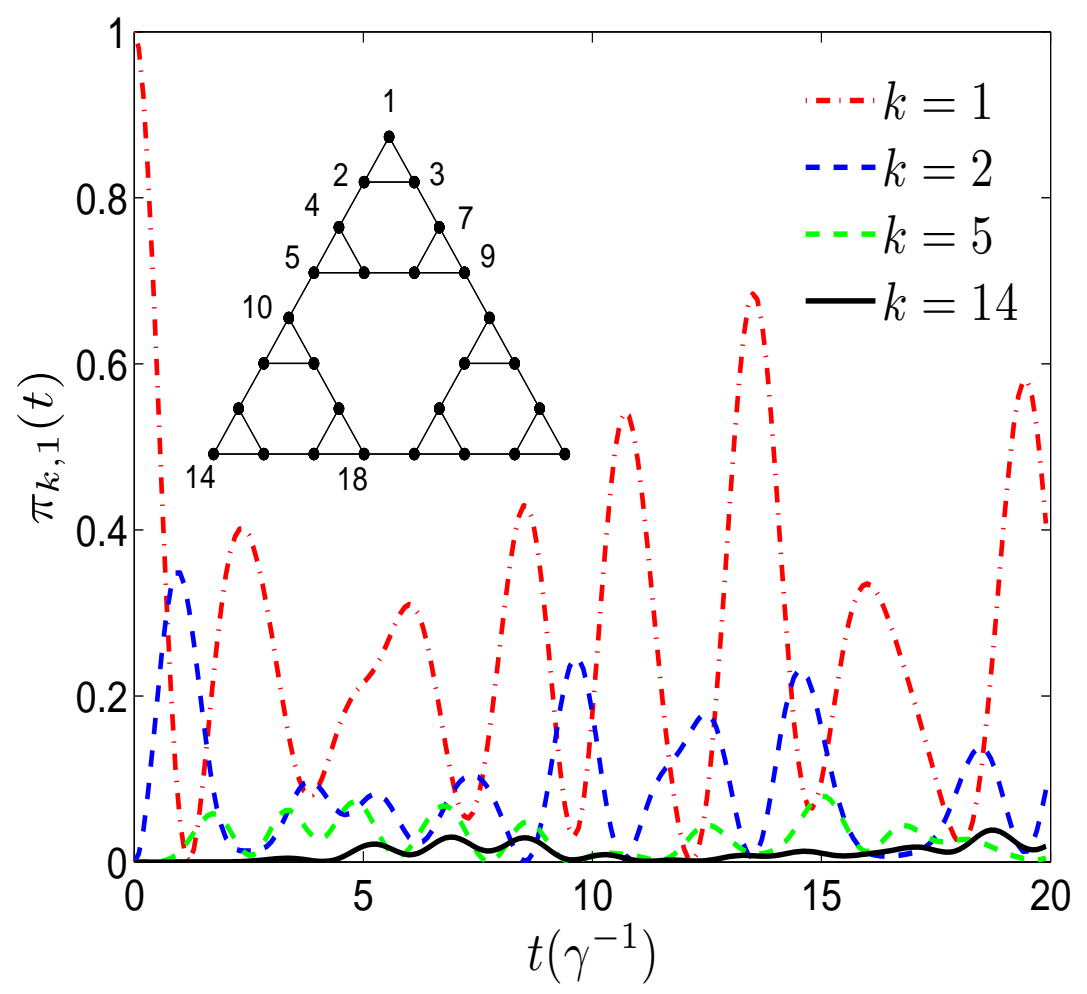

Figure 2. Exact probability $\pi_{k, 1}(t)$ for the CTQW starting from the apex (site $j=1$ ) to reach sites $k=1,2,5$ and 14 . The $k$-sites are the left corners at generations $g=0,1,2$ and 3 , respectively.

the sites 1 and $v_{n}$ increases. This suggests, at least when starting from a main vertex, that the CTQW stays mainly localized at the origin and its neighbourhood.

We corroborate this by looking at the temporal evolution of $\pi_{k, j}(t)$ for the DSG and by comparing it to the $\pi_{k, j}(t)$ pertaining to the CT and the ST of comparable size $\mathcal{N}$. Figures 3, 4 and 5] show 3D pictures of $\pi_{k, j}(t)$ at different moments $t$ (belonging to the short-time regime). On both the $x$ and the $y$-axis, $k$ and $j$ label the nodes of the graph in such a way that at the point $(k, j)$ on the $x y$ plane the value of $\pi_{k, j}(t)$ is presented. At the initial time $t=0$, the transition probability $\pi_{k, j}(t)$ is non-vanishing only on the diagonal, i.e. one has $\pi_{k, j}(0)=\delta_{k, j}$; at later times, $\pi_{k, j}(t)$ spreads out non-uniformly, according to the topology of the substrate. In particular, for the DSG (Fig. 3), a large fraction of $\pi_{k, j}(t)$ stays in a region connected by bonds to the initial nodes; several peaks can be distinguished, whose heights decrease as the chemical distance between the pertaining sites gets larger. For the CT, the pattern representing $\pi_{k, j}(t)$ is even more inhomogenous; as can be inferred from Fig. 4, a quantum particle on the CT is located with very high probability on its initial node (except when starting from the central node) and, for the time scale considered, it is very unlikely to reach nodes outside its starting branch. On the other hand, for the ST (Fig. 5) we notice that the spread of $\pi_{k, j}(t)$ is rapid and regular: apart from possibly partial revival phenomena (see for example the snapshot for $t=3 \gamma^{-1}$ ), the pattern for $\pi_{k, j}(t)$ exhibits very low peaks. 

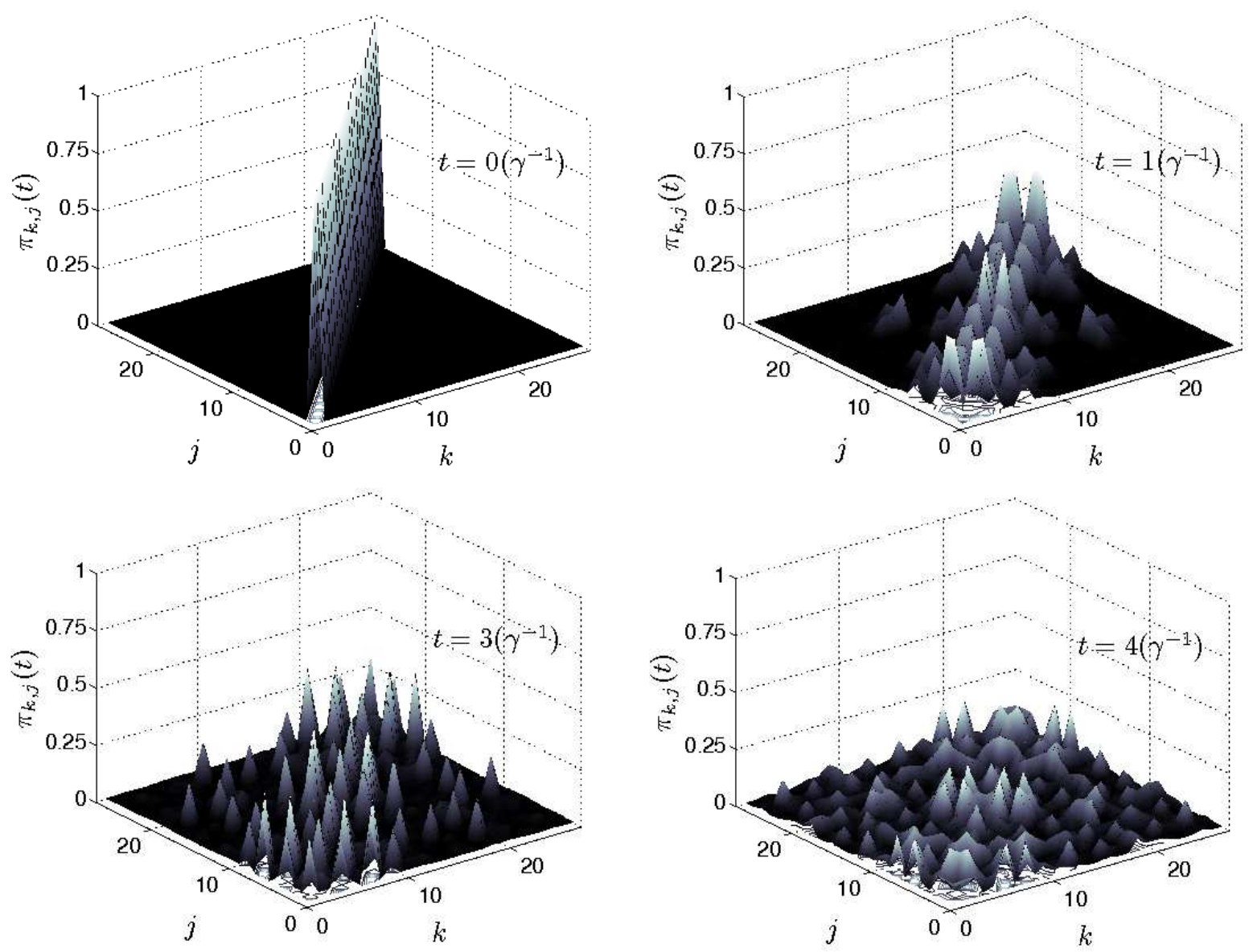

Figure 3. Snapshots of the transition probability $\pi_{k, j}(t)$ at different times $t=0,1,3,4$ (in units of $\left.\gamma^{-1}\right)$ for the Dual Sierpinski Gasket of generation $g=3(\mathcal{N}=27)$.

Indeed, peaks in $\pi_{k, j}(t)$ are a consequence of the constructive interference stemming from reflections at peripheral sites or (in the case of the torus) from the superposition of traveling waves which have crossed the whole (finite) graph.

Finally, Figs. 3, 4 and 5 also highlight the symmetry characterizing the quantum transfer probability, namely that $\pi_{j, k}(t)=\pi_{k, j}(t)$, at all times. This can be derived directly from Eq. 3, recalling that $\mathbf{H}$ is itself symmetric and real. An analogous symmetry also characterizes the classical distribution $p_{j, k}(t)$ for all the cases analyzed here.

\subsection{Average displacement}

The dynamics of quantum particles on non-regular structures has been investigated in several works meant to analyze the quantum dynamics of tight-biding electrons in quasicrystals, in aperiodic and quasi-periodic chains and in random environments [20, 21, 36]. There, the highlighted dramatic deviations from the ballistic behaviour (expected for regular, infinite lattices) range from anomalous to superdiffusion, to decoherence, and even to Anderson localization [37. 

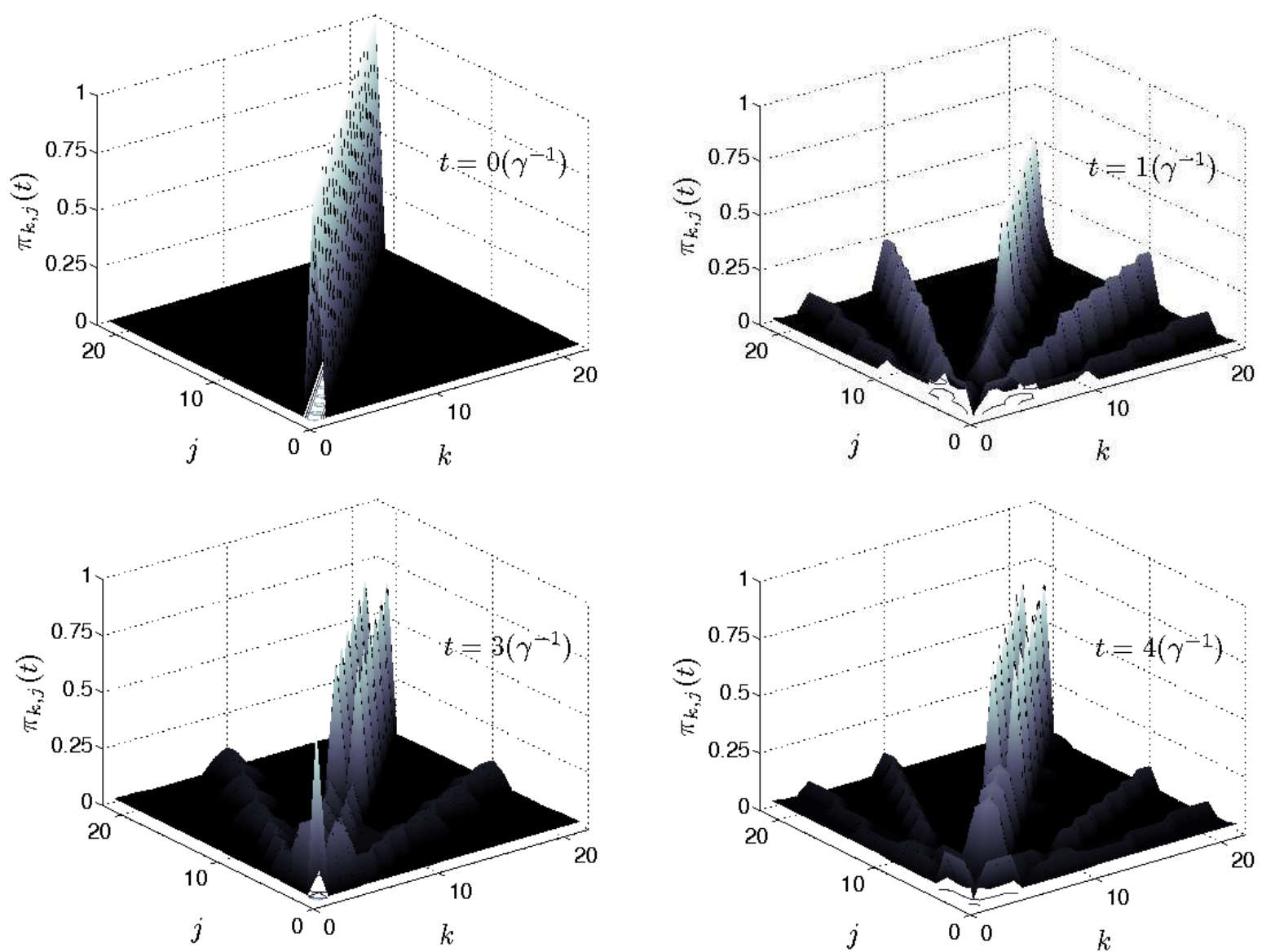

Figure 4. Snapshots of the transition probability $\pi_{k, j}(t)$ at different times $t=0,1,3,4$ for a Cayley Tree of generation $g=3\left(\mathcal{N}=3 \times 2^{g}-2=22\right)$; time is give in units of $\gamma^{-1}$. Notice that the distribution is localized on special couples of nearest-neighbours sites and that also reflection effects appears.

Here, we consider the case in which non-regularity stems from the intrinsic spatial inhomogeneity of the substrate (for DSGs and CTs) and we also study quantum walks on STs which allow to evidence the role of finiteness. From the experimental side, the importance of such factors (spatial inhomogeneities and finiteness of the sample) has been increasingly recognized (see e.g. [38]), so that it is of great interest to understand to what extent quantum transport is influenced by them.

First of all, we notice that the fact that $\pi_{k, j}(t)$ does not attain a stationary distribution also causes the average displacement $\left\langle r_{j}(t)\right\rangle_{q}$ not to necessarily increase monotonically with $t$. Moreover, due to reflection effects, we expect the mean value $\overline{\langle r(t)\rangle}_{q}$ to overestimate the displacement performed by a CTQW that started from a peripheral site. Indeed, one finds for the DSG and the CT that $\overline{\langle r(t)\rangle_{q}}$ is larger than $\left\langle r_{j}(t)\right\rangle_{q}, j$ being any corner of the gasket (see Fig. 6) or any peripheral site, respectively. As for the $\mathrm{ST},{\overline{\langle r(t)\rangle_{q}}}_{\text {trivially equals }}\left\langle r_{j}(t)\right\rangle_{q}$, for all $j$.

As mentioned above, for classical diffusion the average displacement grows continuously from zero to a maximum value $r_{c}$ which, due to equipartition, is just 

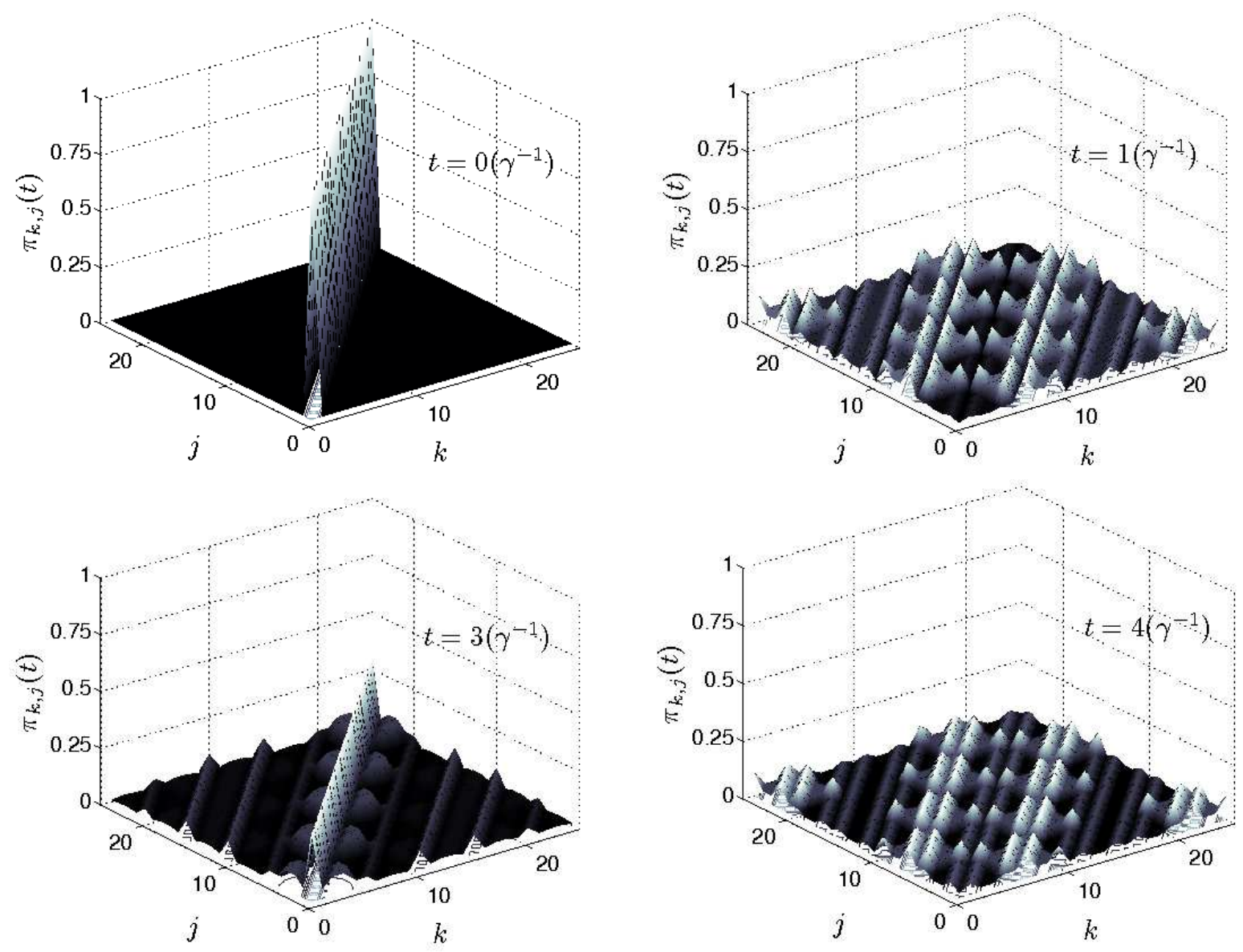

Figure 5. Snapshots of the transition probability $\pi_{k, j}(t)$ at different times $t=0,1,3,4$ (in units of $\left.\gamma^{-1}\right)$ for the Square Torus of linear size $L=5(\mathcal{N}=25)$. Notice that the distribution spreads very rapidly and regularly over the whole structure.

the mean distance among sites:

$$
r_{c}=\frac{1}{\mathcal{N}^{2}} \sum_{k, j=1}^{\mathcal{N}} \ell(k, j) .
$$

Despite of the oscillating behaviour of $\overline{\langle r(t)\rangle}_{q}$, we can obtain an analogous constant value $r_{q}$, around which the average displacement eventually fluctuates, which reads

$$
r_{q} \equiv \lim _{T \rightarrow \infty} \frac{1}{T} \int_{0}^{T} d t{\overline{\langle r(t)\rangle_{q}}}^{T}
$$

Otherwise stated, $\overline{\langle r(t)\rangle}_{q}$ eventually reaches a "stationary regime" in which it fluctuates around a constant value (see Fig. 7).

Of course, $r_{q}$ and $r_{c}$ depend on both the topology and the size of the substrate, and they diverge as $\mathcal{N} \rightarrow \infty$. From the remarks of Sec. 4.1, we expect that quantum interference arising from reflection affects $r_{q}$, making it smaller than $r_{c}$. Indeed, for the CT and the DSG, Fig. 7 clearly shows that $r_{q}<r_{c}$; this is especially apparent for the CT where $r_{c} \approx 8.9$ (calculated from Eq. 15) is nearly four times larger than 


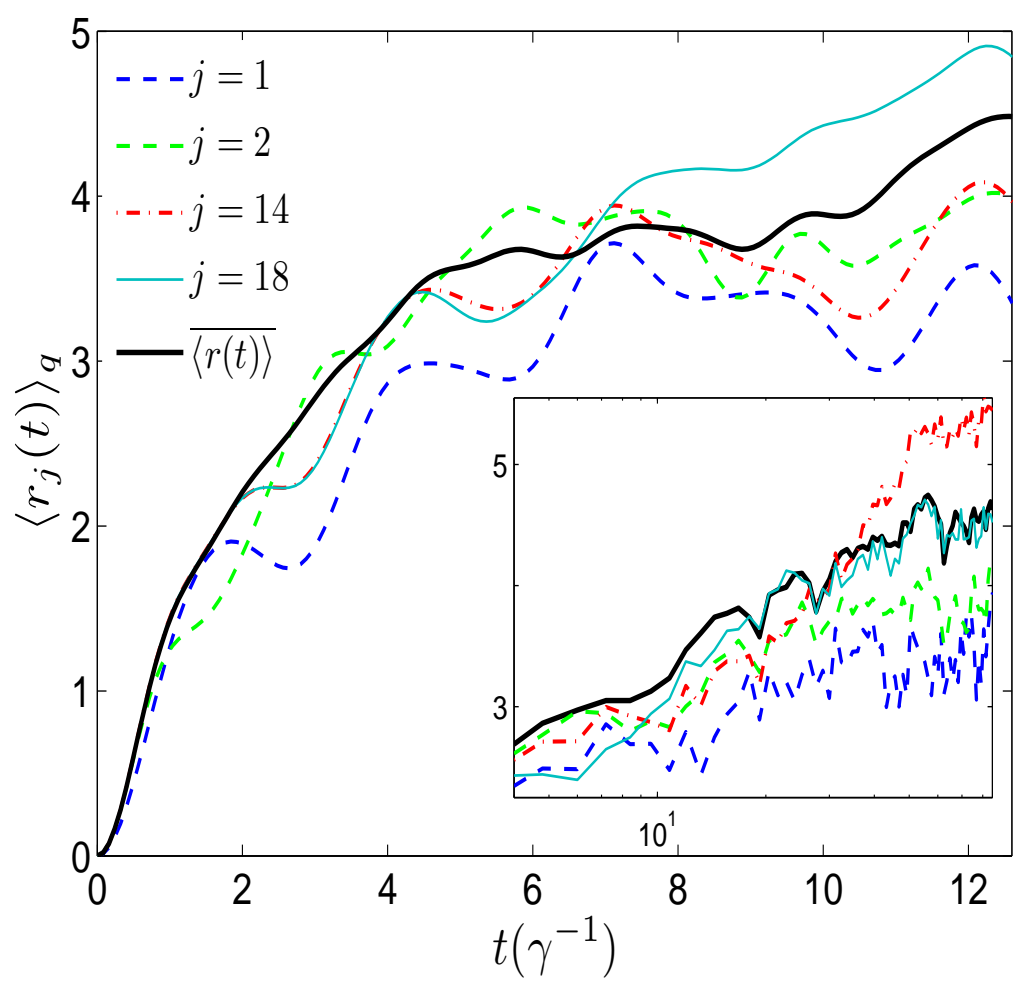

Figure 6. Average displacement $\left\langle r_{j}(t)\right\rangle_{q}$ for a quantum walker which started at the $j$-th site on a DSG of generation $g=5$. The main figure focuses on short times, while in the inset a wider temporal range is considered. Different colours and thicknesses distinguish different initial sites $j$, as shown in the legend; the labeling is the same as in Fig. 2, Notice the appearance of local minima from $t \approx \gamma^{-1}$ onwards.

$r_{q} \approx 2.4$. Conversely, for the ST, where interference only stems from the superposition of waves which have crossed the whole substrate, we find that $r_{c}$ and $r_{q}$ are eventually comparable. Therefore, we expect that on structures endowed with reflecting boundaries (i.e. peripheral nodes of low connectivity), at sufficiently long times, the expectation value of the average distance reached by a quantum particle is strictly smaller than the average distance $r_{c}$ among the nodes.

The classical and the quantum cases are further compared in Fig. 8 which shows the

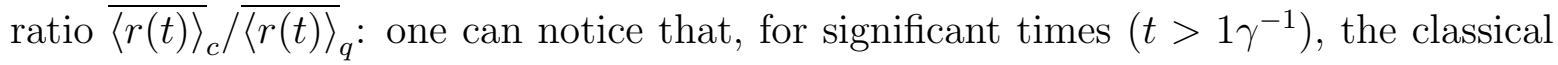
average displacement is strictly lower than the quantum-mechanical one up to time $t \approx 4 \gamma^{-1}, t \approx 9 \gamma^{-1}$ and $t \approx 18 \gamma^{-1}$ for CT, ST and DSG, respectively.

Thus, we can conclude that, on restricted geometries such as those analyzed here, CTQWs can spread faster than their classical counterpart, although the advantage is significant only at relatively short times. Moreover, the spatial homogeneity enhances the speed-up; especially for CTs and, in general, for tree-like structures, the large number of peripheral sites gives rise to localization effects which significantly reduce $r_{q}$.

Finally, we stress that analytical results on the average displacement performed by a quantum particle on discrete structures are rather sparse (see e.g. [16, 39]); in 


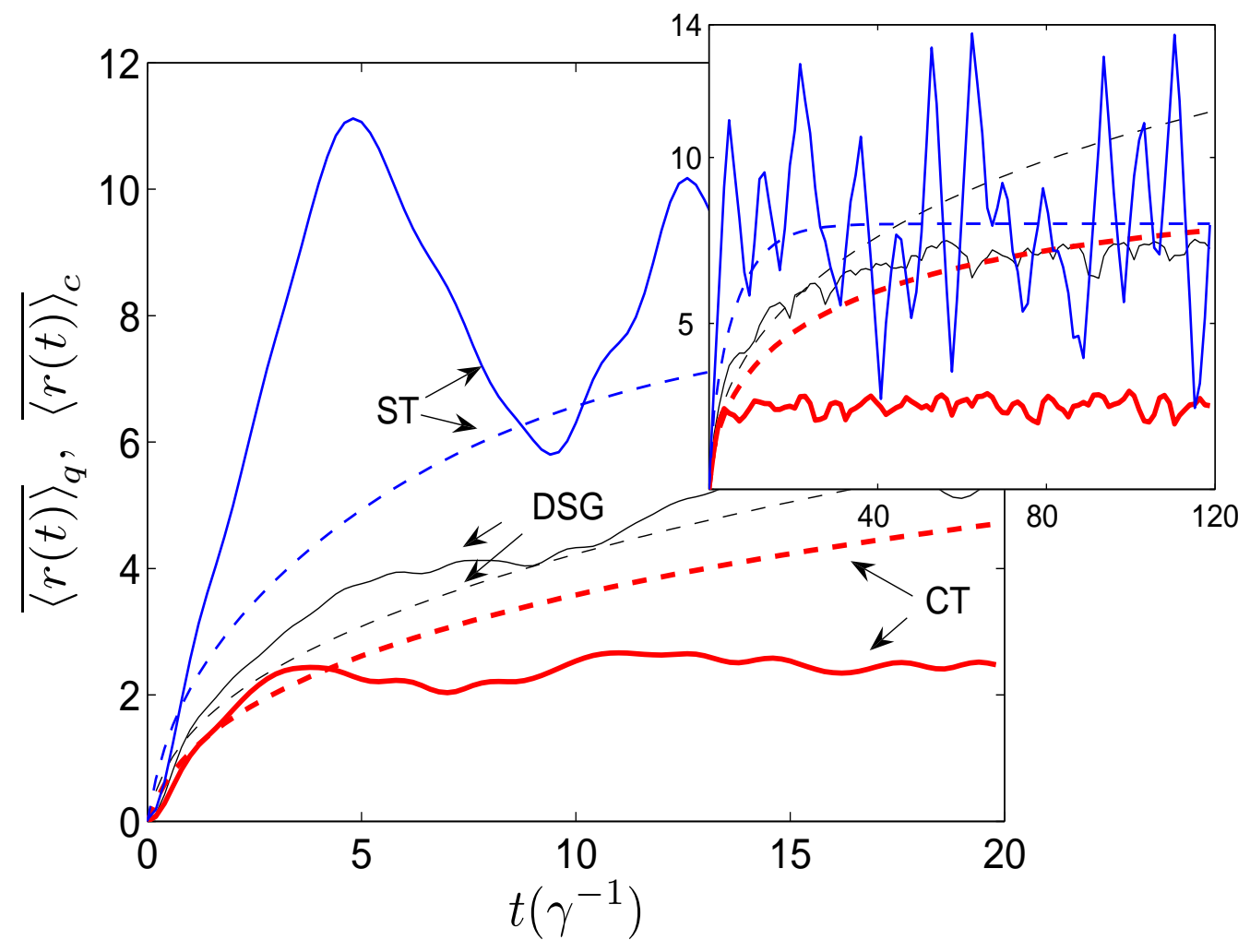

Figure 7. Classical (dashed line) and quantum (continuous line) average displacement for the DSG $(g=5, \mathcal{N}=243)$, the CT $(g=6, \mathcal{N}=190)$ and the ST $(L=16$, $\mathcal{N}=256)$. The main figure focuses on the short-time regime, while the inset also shows the long-time regime.

the Appendix we prove that on infinite $d$-dimensional hypercubic lattices both the average chemical displacement defined in Sec. 2.1 and the Euclidean displacement depend linearly on time and that this kind of behaviour survives, at short times, also for finite lattices.

\subsection{Average return probability}

The average displacement for CTQWs already highlighted some aspects of the role of inhomogeneities for transport processes. Now, we obtain further insights by considering the average return probability.

For the DSG we can get $\bar{p}(t)$ without numerically diagonalizing $\mathbf{L}$, since it only depends on eigenvalues which can be calculated iteratively. Figure 9 displays the averaged probabilities $\bar{p}(t), \bar{\pi}(t)$ and $|\bar{\alpha}(t)|^{2}$ - numerically evaluated from Eqs. 11, 12 and 13, respectively - as a function of time, obtained for $g=5$. The classical $\bar{p}(t)$ decays monotonically to the equipartition value $1 / \mathcal{N}$, while the quantum-mechanical probabilities eventually oscillate around the value 0.7 , which is larger than $3^{-g}$. Although the amplitude of fluctuations exhibited by the lower bound is larger than that of the exact value, the agreement between the two quantities is very good. In 


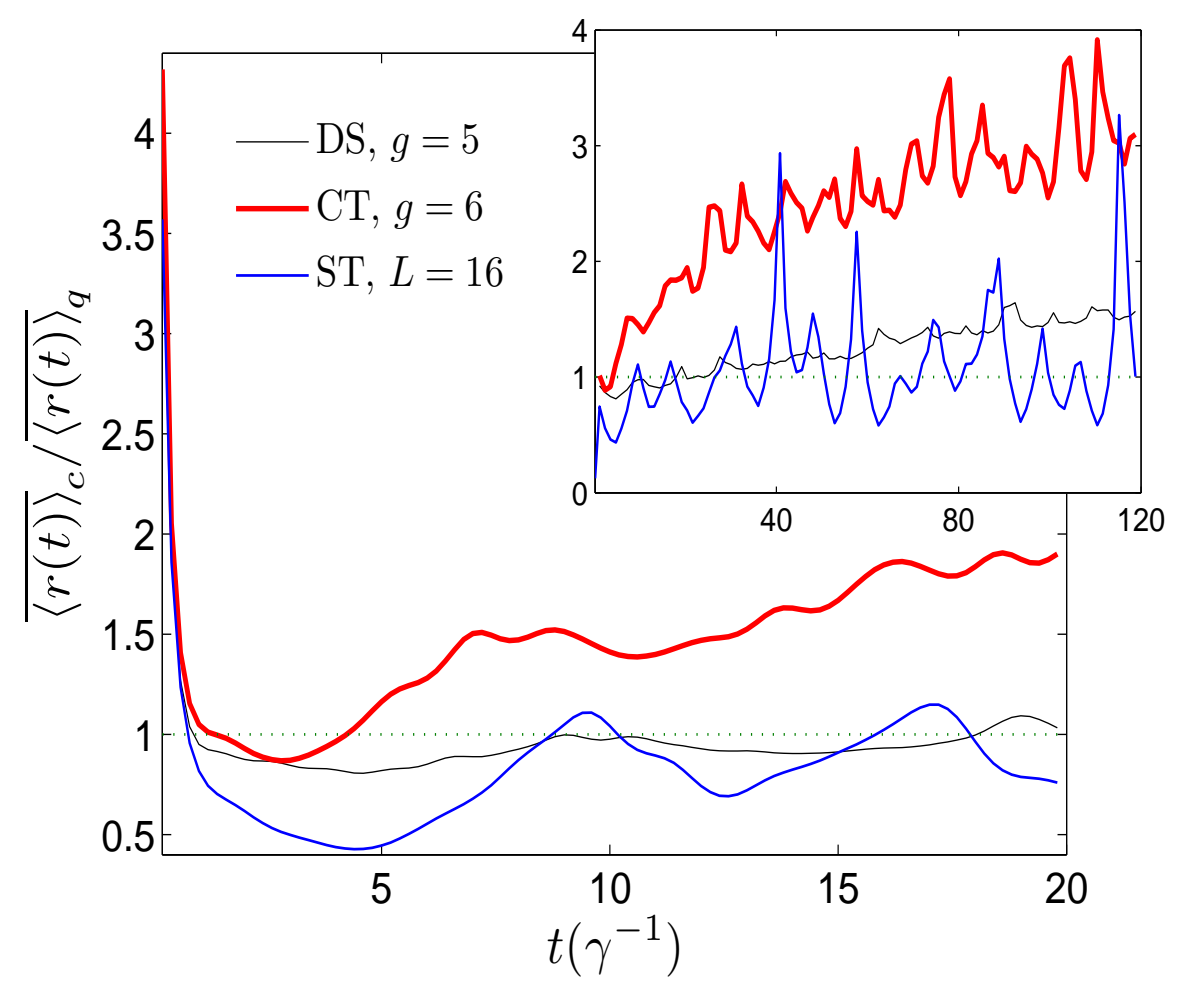

Figure 8. Ratio between classical and quantum average displacement for the DSG $(g=5)$, the CT $(g=6)$ and the ST $(L=16)$, as shown by the legend. The main figure focuses on the short-time, while the inset also shows the long-time regime. In particular, for the square torus, the ratio $\overline{\langle r(t)\rangle}_{c} / \overline{\langle r(t)\rangle}_{q}$ is at first smaller than unity and then it oscillates around unity; the highest peaks are signs of (partial) revivals.

particular, the positions of the extrema practically coincide and the maxima of $\bar{\pi}(t)$ are well reproduced by the lower bound. An analogous behaviour was found also for other graphs, such as square lattices [13], Cayley trees [31] and stars [29]. Notice, however, that for the square lattices the lower bound turns out to be exact while for Cayley trees and for stars it is only an approximation, which, moreover, turns out to be less accurate than what we find here for the DSG.

On short times $\left(t<5 \gamma^{-1}\right)$ it is possible to construct the envelope of $\bar{\pi}(t)$, which depends algebraically on $t$. The exponent is $\approx-0.82$, to be possibly compared with $\tilde{d} / 2 \approx-0.68$ which is the exponent expected classically for the infinite DSG. The decay of the average return probability $\bar{\pi}(t)$ for the ST can be estimated as well: its envelope goes like $t^{-2}$ (classically as $\bar{p}(t) \sim t^{-1}$ ) [13, 30], implying a faster delocalization of the CTQW over the graph.

Interestingly, for the DSG, the overall shape of $\bar{\pi}(t)$ does not depend significantly on the size of the gasket (Fig. 10). In fact, the behaviour of $\bar{\pi}(t)$ is mainly controlled by the most highly degenerate eigenvalues. These do not change when increasing the fractal size (i.e. its generation). These values are: 3 with degeneracy $m_{g}(3)=\left(3^{g-1}+3\right) / 2,5$ 


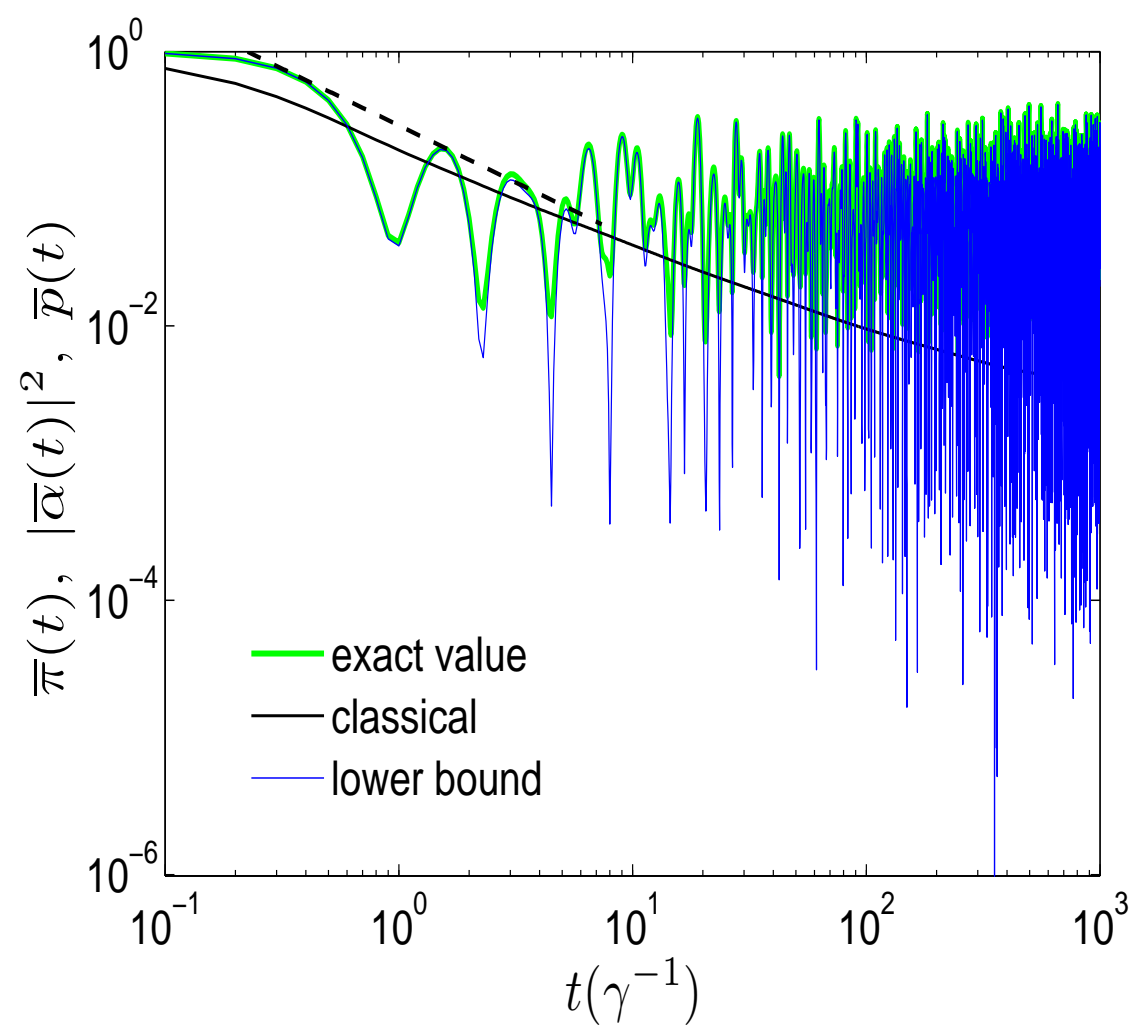

Figure 9. Average return probability $\bar{\pi}(t)$ for the DSG of generation $g=5$ on a log$\log$ scale. The comparison with the classical $\bar{p}(t)$ evidences that the classical random walk spreads more efficiently than its quantum-mechanical counterpart. The dashed line represents the envelope of $\bar{\pi}(t)$.

with degeneracy $m_{g}(5)=\left(3^{g-1}-1\right) / 2$, and $(5 \pm \sqrt{13}) / 2$ with degeneracy $m_{g-1}(3)$, see Sec. 3.

\subsection{Long time averages}

As underlined in Sec. 2, the unitary time evolution does not allow a definite long-time limit for $\pi_{k, j}(t)$. Then, in order to obtain information about the overall spreading of quantum walks, it is advantageous to use the long time average (LTA):

$$
\chi_{k, j} \equiv \lim _{T \rightarrow \infty} \frac{1}{T} \int_{0}^{T} d t \pi_{k, j}(t)=\sum_{n, m} \delta_{\lambda_{n}, \lambda_{m}}\left\langle k \mid \psi_{n}\right\rangle\left\langle\psi_{n} \mid j\right\rangle\left\langle j \mid \psi_{m}\right\rangle\left\langle\psi_{m} \mid k\right\rangle,
$$

where $\delta_{\lambda_{n}, \lambda_{m}}$ equals 1 for $\lambda_{n}=\lambda_{m}$ and is zero otherwise. The LTA of $\bar{\pi}(t)$ follows as

$$
\bar{\chi} \equiv \lim _{T \rightarrow \infty} \frac{1}{T} \int_{0}^{T} d t \bar{\pi}(t)=\frac{1}{\mathcal{N}} \sum_{n, m, j} \delta_{\lambda_{n}, \lambda_{m}}\left|\left\langle j \mid \psi_{n}\right\rangle\right|^{2}\left|\left\langle j \mid \psi_{m}\right\rangle\right|^{2},
$$

for which we obtain a lower bound which does not depend on the eigenvectors [29]:

$$
\bar{\chi} \geq \frac{1}{\mathcal{N}^{2}} \sum_{n, m} \delta_{\lambda_{n}, \lambda_{m}} \equiv \bar{\chi}_{l b}
$$




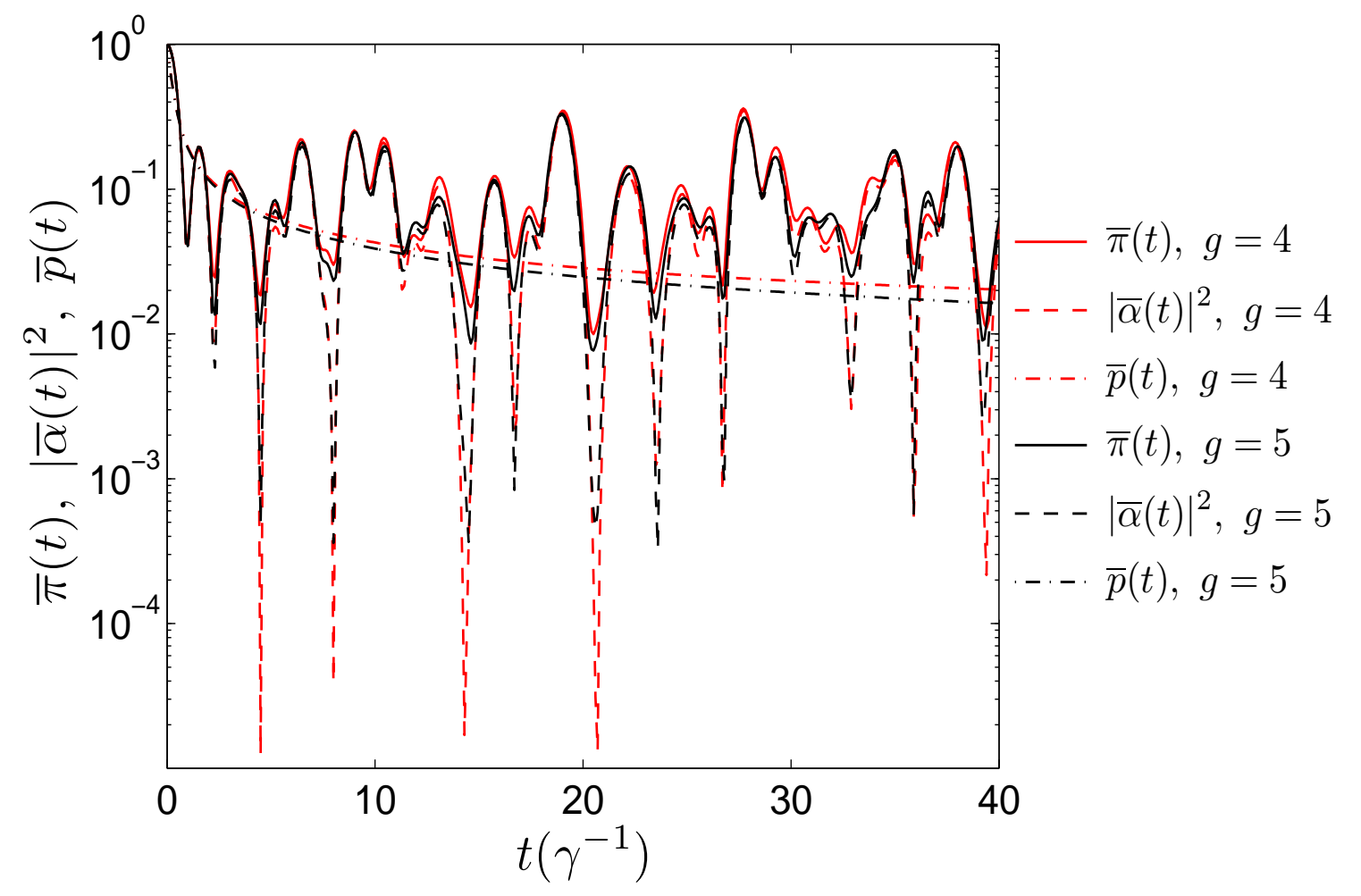

Figure 10. Average return probability $\bar{\pi}(t)$ for the DSG of generation $g=4$ (bright colour) and $g=5$ (dark colour). Its lower bound $|\bar{\alpha}(t)|^{2}$ (dashed line) and the classical $\bar{p}(t)$ (dotted line) are also depicted, as shown by the legend.

We first consider the DSG for which Fig. 11 shows $\chi_{k, j}$ as a contour plot, whose axes are labeled by the nodes $k=1, \ldots, \mathcal{N}$ and $j=1, \ldots, \mathcal{N}$. Bright colours correspond to large, dark ones to small LTAs. First of all, we notice that the LTAs are far from being homogeneous and, hence, are not equipartioned. In particular, the values on the main diagonal are high, meaning that CTQWs have a high LTA probability to be at the starting node.

The inhomogeneity of the pattern mirrors the lack of translation invariance of the DSG itself. For instance, $v$ being the label assigned to any vertex of the main triangle, $\chi_{v, v}$ is a global maximum; off-diagonal local maxima correspond to couples of connected nodes belonging to different minor triangles of generation $g-1$. This allows to establish a mapping between the pattern of $\chi_{k, j}$ and the structure of the relevant DSG. Indeed, as suggested by the white delimiting lines in Fig. 11, the patterns of the LTA distributions exhibit self-similarity.

As for $\bar{\chi}$ and its lower bound $\bar{\chi}_{l b}$, we recall that the former can be calculated numerically, once all eigenvalues and eigenvectors of the Laplacian operator are known (Eq. 18), while for the latter the knowledge of the eigenvalue spectrum is sufficient (Eq. 19). Since the spectrum of the DSG is known, we can calculate $\bar{\chi}_{l b}$ analytically. Recalling the results of Sec. 3, at generation $g$ the spectrum of $\mathbf{L}$ displays $\tilde{\mathcal{N}}$ distinct 

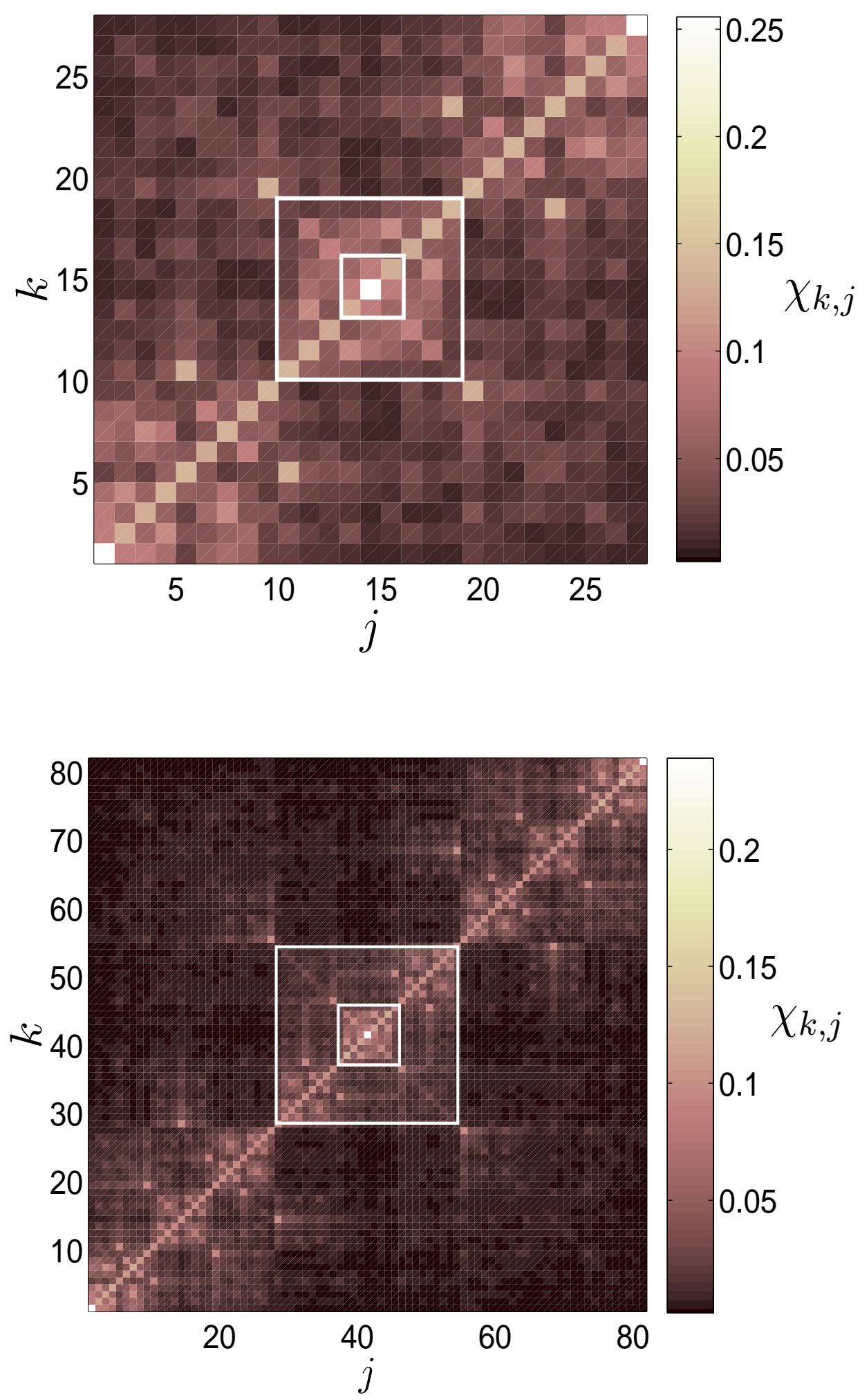

Figure 11. Limiting probabilities for the DSG of generation $g=3$ (top) and $g=4$ (bottom), whose volumes are $\mathcal{N}=27$ and $\mathcal{N}=81$, respectively. The white lines enclose the limiting distributions for gaskets of smaller generations. Notice that the global maxima lay on the main diagonal and correspond to $j=1,14,27$ and to $j=1,41,81$ for $g=3$ and for $g=4$, respectively. 
eigenvalues, where

$$
\tilde{\mathcal{N}}=\sum_{r=0}^{g-1} 2^{r}+\sum_{r=0}^{g-2} 2^{r}+1=3 \times 2^{g-1}-1 .
$$

We call the set of distinct eigenvalues $\left\{\tilde{\lambda}_{i}\right\}_{i=1, \ldots, \tilde{\mathcal{N}}}$. Being $m\left(\lambda_{i}\right)$ the degeneracy of the eigenvalue $\lambda_{i}$, we can write

$$
\mathcal{N}^{2} \bar{\chi}_{l b}=\sum_{n, m=1}^{\mathcal{N}} \delta_{\lambda_{n}, \lambda_{m}}=\sum_{n=1}^{\mathcal{N}} m\left(\lambda_{n}\right)=\sum_{i=1}^{\tilde{\mathcal{N}}}\left[m\left(\tilde{\lambda}_{i}\right)\right]^{2}
$$

Now, we go over to the space of distinct degeneracies, each corresponding to a number $\rho$ of distinct eigenvalues and we get the final, explicit formula

$$
\begin{aligned}
\bar{\chi} \geq & \bar{\chi}_{l b}=\frac{1}{\mathcal{N}^{2}} \sum_{r=0}^{2 g}[m(r)]^{2} \rho(m(r)) \\
= & \frac{1}{\mathcal{N}^{2}}\left\{\sum_{r=0}^{g-1}\left[\frac{3^{g-r-1}+3}{2}\right]^{2} \times 2^{r}+\sum_{r=0}^{g-2}\left[\frac{3^{g-r-1}-1}{2}\right]^{2} \times 2^{r}+1\right\} \\
= & \frac{1}{3^{2 g}}\left[3^{g}\left(1+\frac{3^{g}}{14}\right)+\frac{10}{7} 2^{g}-\frac{3}{2}\right]>\frac{1}{3^{g}} .
\end{aligned}
$$

Interestingly, in the limit $g \rightarrow \infty$, the LTA $\bar{\chi}$ is finite:

$$
\bar{\chi} \geq \lim _{g \rightarrow \infty} \bar{\chi}_{l b}=\frac{1}{14}
$$

and $\bar{\chi}_{l b}$ reaches this asymptotic value from above.

In Fig. 12 we show, as functions of $\mathcal{N}, \bar{\chi}$ and its lower bound, calculated from Eq. 18 and from Eq. 20, For comparison, the same quantities obtained for CTs and STs are also depicted. In the latter case, due to the regularity and periodicity of the lattice [40], the lower bound actually coincides with the exact value. For all cases considered, $\bar{\chi}$ is larger than the equipartion value (given by the dashed line).

The inset of Fig. 12 shows the ratio

$$
\eta(\mathcal{N}) \equiv \frac{\bar{\chi}}{\bar{\chi}_{l b}}
$$

Obviously, the closer $\eta$ is to 1 , the better $\bar{\chi}_{l b}$ approximates $\bar{\chi}$. In this sense, the lower bound calculated for CTs is not as good an approximation to $\bar{\chi}$ as it is for the DSG and for the ST. For the CT, $\bar{\chi}_{l b}$ definitely underestimates, being about half the exact value of $\bar{\chi}$. The quantity $\eta(\mathcal{N})$ may act as a measure of the inhomogeneity of a given substrate. Practically, when dealing with a large sized, sufficiently regular structure, we can get information about the localization of a quantum particle moving on it simply through $\bar{\chi}_{l b}$, thus avoiding the (lengthy) evaluation of the eigenvector set. 


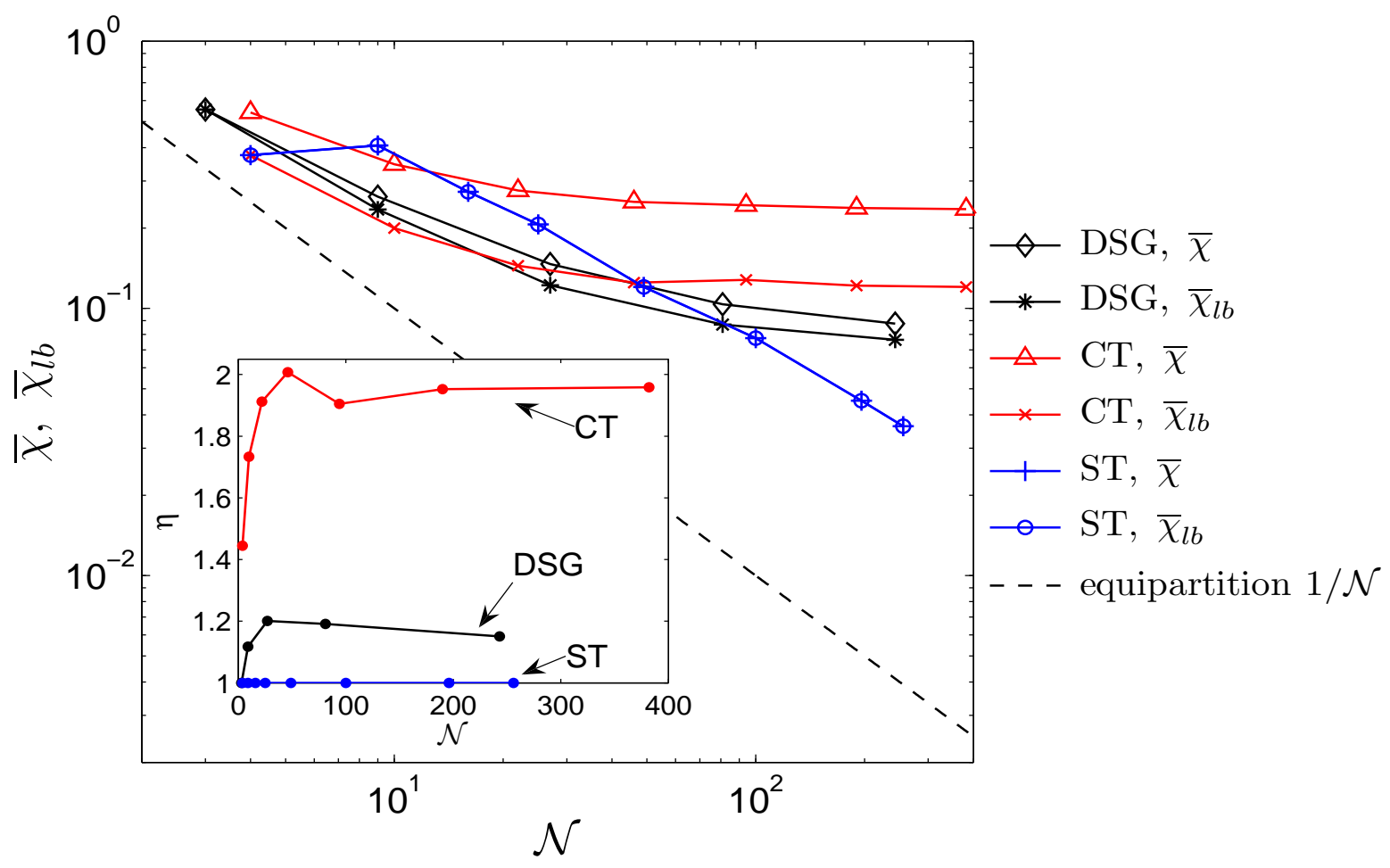

Figure 12. Long time average $\bar{\chi}$ and its lower bound $\bar{\chi}_{l b}$ calculated according to Eq. 20 for the Dual Sierpinski Gasket (black diamonds and stars), the Cayley Tree (red triangles and crosses) and the Square Torus (blue crosses and open circles) versus the size of the structure $\mathcal{N}$; lines are guides for the eye. The dashed line represents the equipartition value $1 / \mathcal{N}$. Inset: ratio $\eta$ as a function of $\mathcal{N}$. Notice that $\eta(\mathcal{N}) \equiv 1$ holds not only for the periodic square lattice, but for all hypercubic lattices.

\section{Conclusions}

We investigated the behaviour of continuous-time quantum walks (CTQWs) on finite discrete structures characterized by different topologies; we considered the Square Torus (ST), the Cayley Tree (CT) and the Dual Sierpinski Gasket (DSG).

The interplay between the quantum-walk dynamics and the underlying topology was deepened by studying, in particular, the temporal evolution of the transfer probability distribution and the ratio $\bar{\chi} / \bar{\chi}_{l b}$ as a function of the substrate size. The latter turns out to be significantly sensitive to the inhomogeneity of the substrate, from which we can infer that lower bound estimates are especially reliable for regular structures.

From an applied, as well as theoretical, perspective, the average displacement of the walker, as a function of time, also plays an important role. This quantity is not only directly related to the transport properties, but it also provides information about how fast the walk explores the underlying structure, allowing an immediate comparison with the classical case. We found that at short times, CTQWs can spread faster than their classical counterparts, although spatial inhomogeneities and finiteness jointly reduce this 
effect. In the Appendix we prove that for infinite $d$-dimensional hypercubic lattices, at long times both the average chemical and the Euclidean displacements depend linearly on time (i.e. the motion is ballistic); for finite lattices this kind of behaviour holds at relatively short times only.

\section{Acknowledgements}

EA thanks the Italian Foundation "Angelo della Riccia" for financial support. Support from the Deutsche Forschungsgemeinschaft (DFG), the Fonds der Chemischen Industrie and the Ministry of Science, Research and the Arts of Baden-Württemberg (AZ: 247532.23-11-11/1) is gratefully acknowledged.

\section{Appendix A. Average chemical displacement on hypercubic lattices}

Here we consider infinite $d$-dimensional hypercubic lattices and, by exploiting their translational invariance, we prove that on them the average chemical displacements of CTQWs, as defined in Sec. 2.1, depend linearly on time. We first focus on the infinite discrete chain, then we consider the generic $d$-dimensional case and finally we analyze the two-dimensional lattice.

For a ring of length $\mathcal{N}$, by exploiting the Bloch states, we have [41]

$$
\alpha_{k, j}(t)=\frac{1}{\sqrt{\mathcal{N}}} \sum_{l} e^{-i \lambda_{l} t} e^{-i l(k-j)}
$$

where $\lambda_{l}$ is the $l$-th eigenvalue of the Laplacian matrix $\mathbf{L}$ associated with the ring. In the limit $\mathcal{N} \rightarrow \infty$ we are allowed to replace the sum over $l$ by an integral, obtaining

$$
\lim _{\mathcal{N} \rightarrow \infty} \alpha_{k, j}(t)=i^{k-j} e^{-i 2 t} J_{k-j}(2 t),
$$

where $J_{k}(z)$ is the Bessel function of the first kind. In the calculation of the transfer probability $\pi_{k, j}(t)$ the phase factor vanishes and we have $\pi_{k, j}(t)=J_{k-j}^{2}(2 t)$, which can be restated as

$$
\pi_{k, 0}(t)=J_{k}^{2}(2 t)
$$

due to the translational invariance of the structure. Clearly (in agreement with $\pi_{k, 0}(t)$ being a probability distribution), one has for all $t$

$$
\sum_{k=-\infty}^{+\infty} \pi_{k, 0}(t)=\sum_{k=-\infty}^{+\infty} J_{k}^{2}(2 t)=J_{0}^{2}(2 t)+2 \sum_{k=1}^{\infty} J_{k}^{2}(2 t)=1
$$

the last equality being based on $J_{k}(z)=(-1)^{k} J_{-k}(z)$ and on Eq. 8.536.3 in [42].

Now, the average chemical displacement of a CTQW which starts from 0 and moves on an infinite chain (subscript $q, 1$ ) follows from Eq. 5 as

$$
\begin{aligned}
\left\langle r_{0}(t)\right\rangle_{q, 1}= & \langle r(t)\rangle_{q, 1}=\sum_{k \in V} \ell(k, 0) J_{k}^{2}(2 t) \\
& =\sum_{k=-\infty}^{\infty}|k| J_{k}^{2}(2 t)=2 \sum_{k=1}^{\infty} k J_{k}^{2}(2 t) .
\end{aligned}
$$


Here, in the first equality we dropped the subscript 0 due to the equivalence between the sites and in the last equality we exploited the symmetry of the Bessel functions, $J_{-k}^{2}(z)=J_{k}^{2}(z)$. Now, recalling the recursion formula Eq. 8.471.1 in [42]

$$
J_{k-1}(z)+J_{k+1}(z)=\frac{2 k}{z} J_{k}(z)
$$

we can write

$$
2 \sum_{k=1}^{\infty} k J_{k}^{2}(z)=z \sum_{k=1}^{\infty}\left[J_{k-1}(z) J_{k}(z)+J_{k}(z) J_{k+1}(z)\right] \equiv z \mathcal{J}(z),
$$

by defining the function $\mathcal{J}(z)$. Hence

$$
\left\langle r_{k}\left(\frac{z}{2}\right)\right\rangle_{q, 1}=z \mathcal{J}(z)
$$

where we put $2 t=z$. The squared Bessel function $J_{k}^{2}(z)$ is almost everywhere positive and the analysis of its zeros allows to state that, for $t>0$, the sum appearing in the left-hand-side of Eq. A.8 is strictly positive; the same holds therefore for $\mathcal{J}(z)$, for which we also notice from Eq. A.7 that $\mathcal{J}(0)=0$. Moreover, through the following recursion formula, Eq. 8.471.2 in 42]

$$
2 \frac{\partial}{\partial z} J_{k}(z)=J_{k-1}(z)-J_{k+1}(z),
$$

it follows by directly differentiating $\mathcal{J}(z)$ and rearranging the terms

$$
\frac{d}{d z} \mathcal{J}(z)=\frac{J_{0}(z)\left[J_{0}(z)+J_{2}(z)\right]}{2}=\frac{J_{0}(z) J_{1}(z)}{z},
$$

where in the last expression we again used Eq. A.6 for $k=1$. The indefinite integral of Eq. A.10 is (see Eq. 5.53 in [42])

$$
\mathcal{J}(z)=z J_{0}^{2}(z)+z J_{1}^{2}(z)-J_{0}(z) J_{1}(z)+C,
$$

as can be simply verified by differentiating Eq. A.11 and using Eqs. A.6 and A.9. Furthermore, since $\mathcal{J}(0)=0$, we have $C=0$.

Therefore, the following, for us fundamental, relation holds:

$$
\sum_{k=1}^{\infty} k J_{k}^{2}(z)=\frac{z}{2}\left[z J_{0}^{2}(z)+z J_{1}^{2}(z)-J_{0}(z) J_{1}(z)\right] .
$$

Now, from Eqs. A.5 and A.12 we get the exact expression for the average chemical displacement

$$
\left\langle r\left(\frac{z}{2}\right)\right\rangle_{q, 1}=z\left[z J_{0}(z)^{2}+z J_{1}(z)^{2}-J_{0}(z) J_{1}(z)\right] .
$$

For large $z=2 t$ (i.e. long times) we can use the expansion (see Eq. 8.451.1 in [42])

$$
J_{k}(z)=\sqrt{\frac{2}{\pi z}}\left[\cos \left(z-\frac{k \pi}{2}-\frac{\pi}{4}\right)+O\left(\frac{1}{z}\right)\right] .
$$

Consequently, inserting Eq. A.14 for $J_{0}(z)$ and $J_{1}(z)$ into A.13, we infer that the long time behaviour of the average chemical CTQW displacement on an infinite chain obeys

$$
\langle r(t)\rangle_{q, 1} \sim \frac{4 t}{\pi}
$$




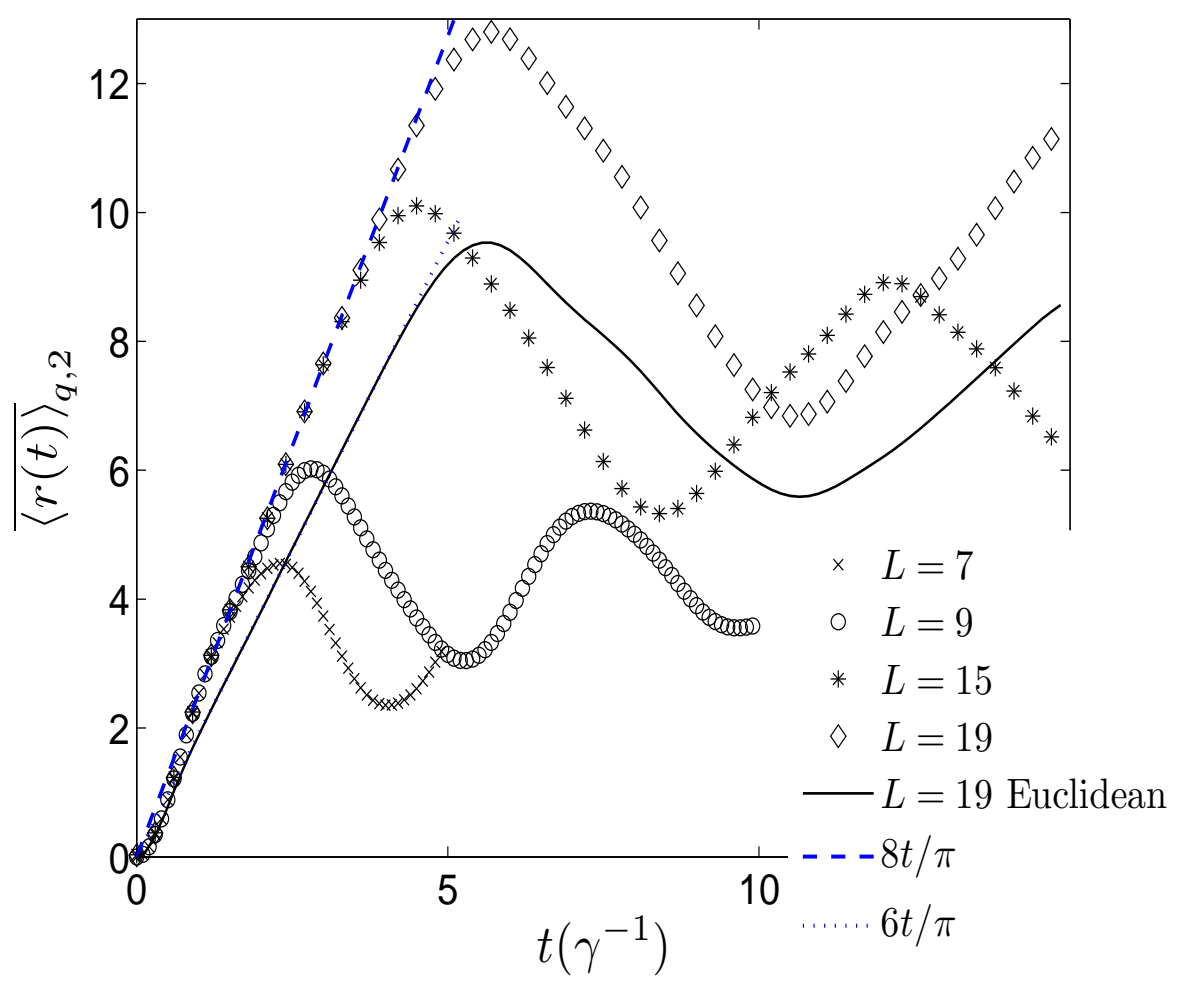

Figure A1. Average chemical displacement $\overline{\langle r(t)\rangle}_{q}$ for the torus calculated according to Eq. 66 (symbols); the line represents the Euclidean average displacement for $L=19$. The dotted and the dashed lines highlight the linear dependence on $t$ exhibited by the average chemical distance and by the average Euclidean distance, respectively.

This result is consistent with findings reported in [39] for the average square displacement.

Let us now consider higher dimensional hypercubic lattices. Again, without loss of generality, we can assume the CTQW to start from the point $\mathbf{0}=(0,0, \ldots, 0)$ so that the chemical distance attained by a walker being at the generic site $\mathbf{k}=\left(k_{1}, k_{2}, \ldots, k_{d}\right)$ is $\ell(\mathbf{k}, \mathbf{0})=\left|k_{1}\right|+\left|k_{2}\right|+\ldots+\left|k_{d}\right|$. Furthermore, on a hypercubic lattice, assuming symmetric conditions in all directions, the probability distribution $\pi_{\mathbf{k}, \mathbf{0}}(t)$ factorizes into the $d$-independent one-dimensional distributions $\pi_{k_{j}, 0}(t)$ :

$$
\pi_{\mathbf{k}, \mathbf{0}}(t)=\pi_{k_{1}, 0}(t) \pi_{k_{2}, 0}(t) \ldots \pi_{k_{d}, 0}(t)=\prod_{j=1}^{d} \pi_{k_{j}, 0}(t) .
$$

Hence

$$
\left\langle r_{\mathbf{0}}(t)\right\rangle_{q, d}=\left\langle\sum_{j=1}^{d}\left|k_{j}\right|\right\rangle_{q, d}=\sum_{j=1}^{d}\left\langle\left|k_{j}\right|\right\rangle_{q, d}=\sum_{j=1}^{d}\left\langle\left|k_{j}\right|\right\rangle_{q, 1}=d\left\langle r_{0}(t)\right\rangle_{q, 1} .
$$

In the last relation we used the fact that $\left\langle\left|k_{j}\right|\right\rangle_{q, d}=\left\langle\left|k_{j}\right|\right\rangle_{q, 1}$ since for each $j$ only the distribution $\pi_{k_{j}, 0}(t)$ matters, the other distributions adding up to a factor of unity each. Hence

$$
\langle r(t)\rangle_{q, d}=d\langle r(t)\rangle_{q, 1} \sim \frac{4 d t}{\pi}
$$


In particular, for the square lattice we have

$$
\langle r(t)\rangle_{q, 2} \sim \frac{8 t}{\pi}
$$

which was used in Fig. A1 (dashed line) to fit data relevant to the average chemical displacement performed by a CTQW on square tori of different (finite) sizes. As can be seen from the figure, the ballistic behaviour also holds for finite lattices, but for relatively short times only: at longer times the finiteness of the lattice starts to matter and the product of Bessel functions in Eq. A.16 ceases to be a good approximation of the transfer probability. When the waves associated with CTQWs have crossed the whole lattice, interference effects start to occur and $\langle r(t)\rangle_{q, 2}$ exhibits a non-monotonic behaviour. From the same figure we also notice that the $O(1 / t)$ contributions of Eq. A.14 get to be negligible for $t>1 \gamma^{-1}$.

In Fig. A1 we also show data for the average Euclidean displacement which displays a ballistic behaviour at short times as well. Indeed, for a hypercubic lattice of arbitrary dimension $d$, the following relation holds (see e.g. [43])

$$
\frac{1}{\sqrt{3}} \ell(\mathbf{k}, \mathbf{j}) \leq\|\mathbf{k}-\mathbf{j}\| \leq \ell(\mathbf{k}, \mathbf{j})
$$

where $\|\mathbf{k}-\mathbf{j}\|$ denotes the Euclidean distance between the lattice points $\mathbf{k}$ and $\mathbf{j}$ chosen arbitrarily. By averaging each term of the previous equation with respect to the transfer probability $\pi_{\mathbf{k}, \mathbf{j}}(t)$ (we can again exploit the translational invariance of the substrate and fix $\mathbf{j}=\mathbf{0}$ ), we find that the average Euclidean distance also scales linearly with time with a multiplicative factor bounded between $4 d /(\sqrt{3} \pi) \approx 2.31 d / \pi$ and $4 d / \pi$. In particular, for the square torus of size $L=19$ considered in Fig. A1, we find that at relatively short times the average Euclidean distance scales as $6 t / \pi$.

\section{References}

[1] Kempe J 2003 Contemp. Physics 44307

[2] Sanders BC, Bartlett SD, Tregenna B and Knight PL 2003 Phys. Rev. A 67042305

[3] Lahini Y, Avidan A, Pozzi F, Sorel M, Morandotti R, Christodoulides DN, and Silberberg Y 2008 Phys. Rev. Lett. 100013906

[4] Dür W, Raussendorf R, Kendon VM and Briegel H-J 2002 Phys. Rev. A 66052319

[5] Côté R, Russell A, Eyler EE and Gould PL 2006 New J. Phys. 8156

[6] Zou X, Dong Y and Guo G 2006 New J. Phys. 881

[7] Ryan CA, Laforest M, Boileau JC and Laflamme R 2005 Phys. Rev. A 72062317

[8] Mülken O, Blumen A, Amthor T, Giese C, Reetz-Lamour M and Weidemüller M 2007 Phys. Rev. Lett. 99090601

[9] Aharonov Y, Davidovich L and Zagury N 1993 Phys. Rev. A 481687

[10] Farhi E and Gutmann S 1998 Phys. Rev. A 58915

[11] Strauch FW 2006 Phys. Rev. A $74030301(\mathrm{R})$

[12] Štefaňák M, Jex I and Kiss T 2008 Phys. Rev. Lett. 100020501

[13] Volta A, Mülken O and Blumen A 2006 J. Phys. A 3914997

[14] Mülken O, Pernice V and Blumen A 2007 Phys. Rev. E 76051125

[15] ben-Avraham D and Havlin S, Diffusion and Reactions in Fractals and Disordered Systems, (Cambridge University Press, 2001). 
[16] J. Vidal, R. Mosseri and J. Bellissard 1999 J. Phys. A 322361

[17] Williams CP 2001 Computing in Science and Engineering 3, 244

[18] Ambainis A 2004 SIGACT News 3522

[19] Magniez F, Nayak A, Roland J and Santha M, in Proceedings of ACM Symposium on Theory of Computation (STOC'07) (ACM Press, New York, 2007), p.575

[20] Yin Y, Katsanos DE and Evangelou SN 2008 Phys. Rev. A 77022302

[21] Yuan HQ, Grimm U, Repetowicz P and Schreiber M 2000 Phys. Rev. B 6215569

[22] Graph Theory, Combinatorics, and Applications, Vol. 2, Ed. Y. Alavi, G. Chartrand, O.R. Oellermann, A.J. Schwenk, Wiley, 1991.

[23] Di Vincenzo DP 1995 Science 270255

[24] Childs AM and Goldstone J 2004 Phys. Rev. A 70022314

[25] Mülken O, Volta A and Blumen A 2005 Phys. Rev. A 72042334

[26] Weiss GH, Aspects and Applications of the Random Walk, (North-Holland Press, 1994)

[27] Aharonov D, Ambainis A, Kempe J and Vazirani U, in Proceedings of ACM Symposium on Theory of Computation (STOC'01) (ACM Press, New York, 2001), p.50.

[28] Aarão Reis FDA 1995 J. Phys. A 286277

[29] Mülken O 2007 arXiv:0710.3453

[30] Mülken O and Blumen A 2006 Phys. Rev. E $\mathbf{7 3} 066117$

[31] Mülken O, Bierbaum V and Blumen A 2006 J. Chem. Phys. 124124905

[32] Konno N 2006 Quantum Probability and Related Topics 9, 2287

[33] Konno N 2006 Int. J. Quant. Inf. 4, 61023

[34] Cosenza MG and Kapral R 1992 Phys. Rev. A 461850

[35] Blumen A and Jurjiu A 2002 J. Chem. Phys. 1162636

[36] Cerovski VZ, Schreiber M and Grimm U 2005 Phys. Rev. B 72054203

[37] Anderson PW 1958 Phys. Rev. 1091492

[38] Monastyrsky MI, Topology in Condensed Matter, Springer Series In Solid-State Sciences, SpringerVerlag Berlin Heidelberg 2006

[39] Katsanos DE, Evangelou SN and Xiong SJ 1995 Phys. Rev. B 51895

[40] Blumen A, Bierbaum V and Mülken O 2006 Physica A 37110

[41] Mülken O and Blumen A 2005 Phys. Rev. E 71036128

[42] Gradshteyn IS and Ryzhik IM, Table of Integrals, Series and Products, Academic Press, Inc. 1965.

[43] Searcóid MO, Metric Spaces, Springer-Verlag, London 2007. 